\title{
RCT - a tool for continuous road pavement diagnostics
}

\author{
Marcin Staniek ${ }^{1, *}$ \\ ${ }^{1}$ Silesian University of Technology, Faculty of Transport, Krasińskiego 8, 40-019 Katowice, Poland
}

\begin{abstract}
The paper addresses a road pavement diagnostics solution created as a sub-module of the S-mileSys platform developed as a part the S-mile project, implemented under the "Sustainable Logistic and Supply Chain" competition of the ERANET Transport III programme. The S-mileSys solution was conceived as a means to support transport companies and their clients in planning, organisation and delivery of freight transport related services, but also to support local authorities and road administration bodies in matters related to road infrastructure maintenance and traffic management within cities or agglomerations. In principle, as typical transfers are made in connection with freight transport, the process of continuous road pavement monitoring and assessment is performed in a manner which does not involve interference of either vehicle drivers or employees of transport companies or logistics centres. The measuring system's operation is essentially based on identification of values of linear accelerations recorded while vehicles traverse the road network. ICT solutions are used to send characteristics describing the vehicle motion dynamics, determined by the road pavement condition, to the S-mileSys system's server, where, based on analysis of linear acceleration signals in a three-dimensional description of space and by taking GPS positioning into consideration, the current road pavement condition is estimated.
\end{abstract}

\section{Introduction}

Contemporary road pavement diagnostics requires application of highly specialised measuring systems to perform assessment of technical and operating parameters of road infrastructure. A set of solutions jointly referred to as Pavement Management System (PMS) are designed to support road administration bodies. According to PMS guidelines, the parameters subject to assessment under the measurement procedure include the following [1]:

- load capacity of structures,

- longitudinal evenness,

- transverse evenness,

- skid resistance properties,

- surface properties.

The pavement load capacity assessment, including identification of sections characterised by inferior or even completely depleted load capacity, is usually conducted by measuring pavement deflection under load. For these purposes, one typically uses beam deflectometers [2] or Falling Weight Deflectometers [3], and where the latter is the case, load is applied by a mass free falling from a predefined height. The latest measurement solutions include laser Traffic Speed Deflectometers based on laser scanning used to measure vertical displacement caused by the test vehicle weight [4]. Structure surveying by means of test boreholes which must be developed in road pavement is gradually being replaced by non-intrusive methods. There are entire radar systems, known as Ground Penetrating Radars, developed by application of the electromagnetic wave propagation theory. They enable identification of the arrangement of structural road courses as well as assessment of inner voids in individual pavement layers [5].

The solutions one can also use to analyse longitudinal and transverse evenness include laser surface analysers and planographs. The former are characterised by particularly high measuring accuracy and simplicity of the measurement procedure. They make it possible to compare results with a theoretical road profile. It should also be added that using surface analysers to measure road evenness causes no inconvenience to other road traffic participants [6]. Based on a real-life profile, diverse longitudinal evenness indicators are determined, including IRI for computational road sections.

One can estimate skid resistance properties of a road, i.e. its friction coefficient, by several measurement methods, one of which consists in direct measurement of the forces affecting a standard test wheel installed in a measuring device once the wheel has been set in parallel and at a constant pre-set angle to the direction of motion. Some model measuring solutions include the SCRIM test vehicle [7] as well as the SRT-3 measuring set [8]. An alternative solution is testing of the road pavement texture by means of pre-calibrated sand spread over the road surface, where the assumed sand quantity and its spread determine the roughness of the pavement examined [9]. The visual solution based on application of light patterns and used to estimate the level of road pavement roughness is presented in [10]. There are also tools to test of the road pavement texture basing on laser scanning $[11,12]$.

Assessment of superficial properties of road pavements includes detection and surveying of:

- alligator-type cracks,

* Corresponding author: marcin.staniek@polsl.pl 
- single cracks,

- embedded patches,

- surface-applied patches,

- potholes,

- pavement bleeding,

- pavement raveling.

The available solutions used for identification of the above defects are primarily based on image processing and recognition technologies [13]. One should note that visual methods require video input material of high quality, including a wide range of the pixel intensity function, homogeneous illumination or low image noise. It is possible to maintain high quality of the input material through calibration of the image recording system, noise filtering and correction of optical distortions in road pavement images [14].

Several parameters of the road infrastructure condition description can be identified in a single series of measurements owing to highly specialised test vehicles, also referred to as mobile road laboratories. One of them is the ARGUS solution [15]. Test vehicles such as this allow for assessment of longitudinal and transverse evenness as well as identification of pavement surface defects. They additionally take sequential photographs of the road lane space for purposes of recording and verification of technical conditions of road infrastructure elements.

The literature of the subject also mentions road pavement assessment solutions that are based on analysis of linear accelerations recorded as a vehicle moves in the road network. According to the basic multi-channel approach of this method, the data logger unit records accelerations induced by vibrations of the driven wheel moving on the road section subject to examination, while it is loaded by the mass applied. What the analysis of vehicle motion dynamics description requires is to filter out the noise emerging as an outcome of the mass system damping. Model solutions based on this approach have been described in papers [16,17].

Presented solutions in the diagnostics of road pavement are advanced measurement systems requiring trained inspectors to carry out the measurement procedure. The systems have high costs of their construction and execution of the measurements. Under the efforts undertaken in the S-mileSys platform building project, as a means to identify and describe road pavement condition, the author of this article proposed a method for recording of the vehicle motion dynamics and a method for analysing the recorded linear acceleration signals in a three-dimensional space description. The solution thus developed stems from field surveys as well as a review of the available solutions for identification and assessment of road pavement condition, having analysed the complexity of its structure and taken the simplicity of the measurement procedure into consideration. What proved to be of primary importance for the solution in question was the set of pre-assumed priorities, namely the operation which did not entail the driver's involvement, non-intrusive installation of the measuring device onboard of the vehicle as well as non-interference in the vehicle control signals.

\section{Characteristics of the S-mileSys platform}

What had determined the attempt to develop the S-mileSys platform was the EU's premises and policies for shaping and developing sustainable logistics, including the supply chain support for the freight transport sector [18]. The primary goals assumed for the solution being developed included efficient transport route planning over the first/last mile by taking innovative route optimisation criteria into account, including identification and selection of eco-friendly and community-oriented solutions in freight transport related services. The foregoing is assumed to support local authorities in making transport system management related decisions for the given area and to expand the potential of transport companies [19].

The idea behind the S-mileSys platform is pursued under the international project entitled "Smart platform to integrate different freight transport means, manage and foster first and last mile in supply chains (S-mile)", cofinanced under the "Sustainable Logistics and Supply Chains" competition within the framework of the ERANET Transport III programme. An international consortium of contractors incorporated for that purpose consists of the following institutions that represent three countries: SAITEC, Factor CO2 and DeustoTech from Spain, the Silesian University of Technology from Poland and Plus One Minus One from Turkey.

According to the S-mile project assumptions, the S-mileSys platform has been devised as a multi-server IT system, based in terms of both operation and integration on client-server solutions along with a web-based user interface. The S-mileSys platform's architecture, including groups of stakeholders related to individual tools and platform modules, has been shown in Fig. 1.

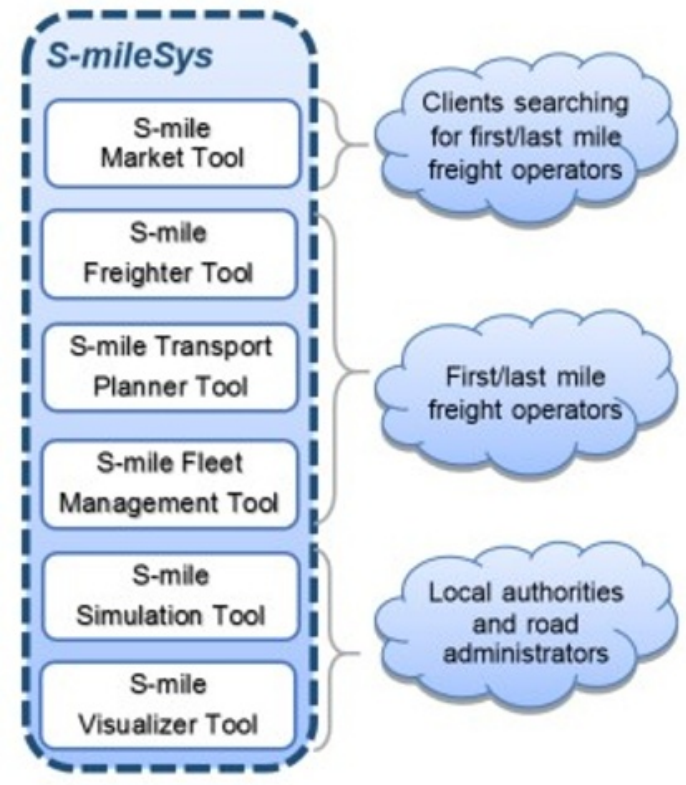

Fig. 1. Architecture of the S-mileSys platform 
The S-mileSys platform development project comprises design and implementation of six main tools/modules. S-mile Transport Planner Tool (1) is the key component - a tool that enables planning and organisation of transport services by collecting and distributing goods along a single route, optimisation of routing according to basic (distance, time, cost) as well as innovative (environmental, social and infrastructural) criteria, but also updating of these routes by taking road traffic parameters into consideration. From the perspective of the subject of this article, the most important one is the goods transport route planning criterion from the perspective of road infrastructure condition. This means that one should strive to minimise road network degradation due to heavy vehicle traffic by setting their routes outside network sections in poor condition. A tool which has been designed particularly with local authorities in mind, including road infrastructure administrators, is S-mile Visualizer Tool (2), which enables monitoring of road pavement condition and road traffic parameters and which makes it possible to obtain information about transport service routes and emission of harmful substances in selected city or agglomeration districts. Another tool in the package is Smile Simulation Tool (3), intended to enable public institutions and companies operating in the environmental protection sector to conduct comprehensive simulation and assessment of the environmental impact of various motivational, organisational or fiscal initiatives aimed to exert influence on how city authorities implement their environmental, economic, infrastructural or social policies against freight transport.

S-mile Freighter Tool (4) is intended to support transport companies by enabling them to conduct continuous monitoring of their dispatched vehicle fleet and to review fleet availability data, but also ensuring communication between drivers and in-house dispatchers. It covers diverse ICT solutions, including custom-made mobile applications designed to be used for road pavement diagnostics, among other purposes. S-mile Fleet Management Tool (5) is a solution that fosters efficient transport planning, enables carriers to generate reports for purposes of calculations of total freight transport costs as per defined attributes taking into consideration the pre-set transport routes, carriers' needs and restrictions imposed by local road network administrators. Integration of companies which provide transport services with clients who seek such companies is the function of S-mile Market Tool (1) which - with considerable simplification - could be compared to a typical freight exchange.

According to the assumptions adopted as the grounds for the S-mileSys platform development, data describing the vehicle motion dynamics, taking its location into account, are to be rendered by transport companies which have acquired this system and which provide transport services in the area of the chosen city or agglomeration. What these companies receive in return is a form of support comprising implementation of innovative tools, i.e. S-mile Freighter Tool and S-mile Fleet Management Tool, intended for numerous purposes, including to support drivers and dispatchers at the vehicle fleet management centre, while they simultaneously transfer data considered insensitive by these companies to local authorities for the sake of effective description of the transport system condition.

\section{System of road pavement condition diagnostics}

According to the main assumption underlying the S-mileSys platform specification, carriers' vehicles are equipped with mobile devices ensuring connection between the driver transporting cargo and the dispatcher at the transport company's headquarters. Such a communication channel offers additional features, including the capacity to navigate the driver along the route, monitor transport services, track the vehicle's location and operating parameters, status of the cargo as well as the driver's working time and driving style.

Besides functions typically dedicated to urban freight transport, mobile devices with the application developed under the S-mile project on board also provide feedback capabilities. They record diverse data and transfer them to one of S-mileSys servers, including data that describe the current technical condition of road pavements, assuming the form of characteristics of changes to the dynamics of the given vehicle as it traverses the chosen area of the road network.

\subsection{Mobile device for data acquisition}

The solution proposed for purposes of data acquisition is a mobile device, namely a typical smartphone or tablet running on the Android operating system's KitKat 4.4 release, or higher, featuring the RCT mobile application developed and implemented under the S-mile project. According to the assumptions adopted for the RCT mobile application, the mobile devices on which the application is to be installed must feature the MEMS, GPS and GSM modules. The device's operating principle consists in high-frequency recording of linear accelerations acquired from the MEMS module, linked with individual locations identified by the GPS receiver and stored in the device memory as an output file containing series of data recorded in line with a pre-defined standard. Moreover, in order to ensure communication between the device on board of the vehicle and the transport company's system, the device must be equipped with a GSM module. Following the transport service provision, the output file is sent from the RCT mobile application to one of the RCT server system's servers via the FTP protocol. Additionally, in order to secure the transmission, i.e. to confirm completeness of data transfer to the server, the mobile device generates an acknowledgement signal (ACK) the receipt of which is confirmed in the S-mileSys system.

The RCT mobile application can perform the data acquisition function in two operating modes: remote and manual. In the remote mode, the application is controlled by a higher rank application installed on the mobile device, supporting the driver's activity. In this mode, the RCT mobile application requires no intervention from the 
driver. The manual mode makes it possible to define specific identifiers to describe the route being covered and the frequency of readouts from the required modules. The process used to describe the vehicle motion dynamics is independent of the chosen operating mode. The graphical user interface available in the manual mode of the RCT mobile application has been depicted in Fig. 2.
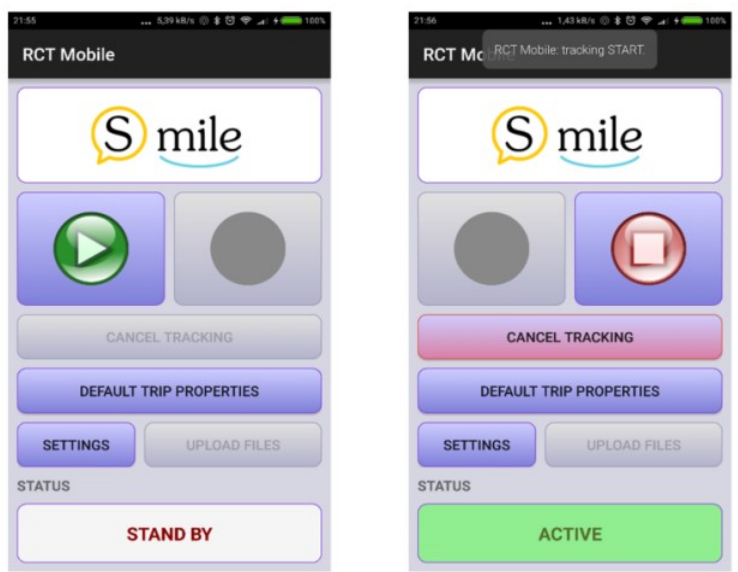

Fig. 2. RCT mobile application

The main application window allows the user to choose between six available functions and contains the application mode status field. Respective buttons/functions start and stop the data acquisition procedure and enable instantaneous data recording interruption in unplanned situations, e.g. in the event of an application failure. The Default trip properties function makes it possible to define route identifiers, while pressing the Settings button enables the user to set such parameters as frequency of data readout from the MEMS and GPS modules as well as the readout precision. The Upload files button performs an auxiliary function, making it possible to resume the process of data transmission to the server in cases of unplanned data transmission interruption.

Regardless of the application operating mode, the outcome is an output file containing the following header of the transport service route description:

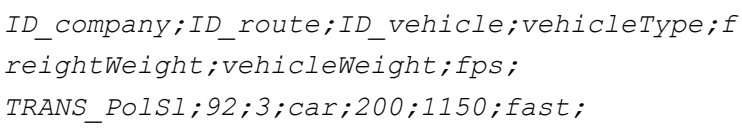

as well as the main file body, i.e. a series of data of the recorded vehicle dynamics in the following format (sample data segment):

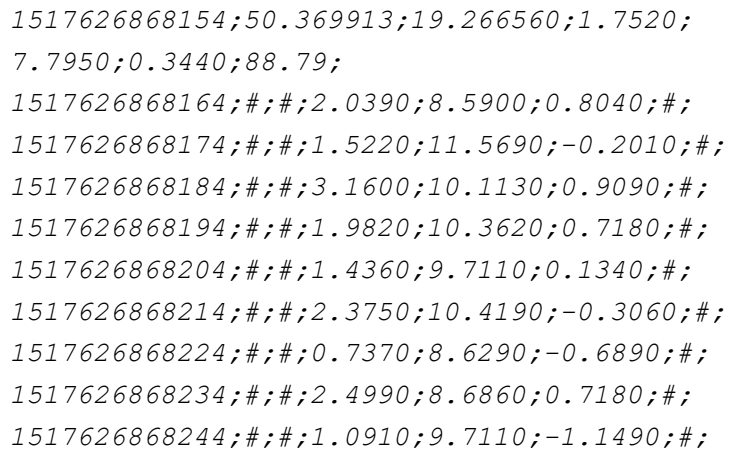

The output file produced by the RCT mobile application is sent to RCT server where the data series is verified, and the final outcome of the process is estimation of a number of indices, including those of the road pavement condition assessment.

\subsection{Data series verification and processing}

The process of data series verification and processing is performed in the following consecutive steps. Once the data receipt is confirmed on RCT server, the first stage of the process begins, namely data verification. What the server checks is the data series continuity and correctness of the values defining both accelerations and locations according to the verification of assignment of the acquired data and the pre-defined range of values. Discontinuity of the data series in the function of time as well as deviations of temporary values from the mean initiate the data set filtering procedure. It is also possible that an entire output file containing the recorded vehicle motion dynamics data is rejected. The second stage consists in mapping the route recorded as a series of GPS coordinates over a set of the OSM map sections. It is characterised by a complex mapping algorithm which - for purposes of the process applies the criterion of the GPS track coverage by routes of OSM sections, the criterion of conformity between the direction of the resultant vector of the GPS track and the OSM section direction as well as the criterion of continuity of the GPS track mapping assigned to successive OSM sections.

Bearing in mind the assumptions made with regard to how the S-mileSys platform should function and the goals pursued while developing the platform, two types of sections were introduced: segments and parts. For the sake of the freight transport route planning, a segment was defined as a section between consecutive GIS coordinates on a map, characterised by diverse length depending on the road network specificity. For purposes of the road pavement condition description conforming with the current requirements of PMS systems, a part was defined as a section of fixed length defined by the system administrator.

Once a route extracted from the output file is assigned to individual OSM sections, it is possible to establish the road pavement condition assessment index.

\subsection{Estimating the road pavement assessment index}

The road pavement assessment index performs two functions on the S-mileSys platform. Firstly, it is an element of the road infrastructure status description intended for local authorities, including road administration bodies. Secondly, it is an element of the freight transport route planning algorithm which enables bypassing of road sections in poor condition, intended for transport companies. However, irrespective of the function it performs, the road pavement condition assessment index assumes a value ranging between 0 and 1 , where 0 corresponds to a road section of good condition (technical class A as per the applicable guidelines [20]), 
while 1 defines a section of poor condition (technical class $\mathrm{D}$ as per the applicable guidelines [20]).

The $\delta_{\text {id }}$ part road pavement condition assessment index for a pre-set road section of id part is estimated with reference to a single transfer made with a vehicle equipped with a device having the RCT mobile application on board according to the following relationship:

$$
\delta\left(d_{\text {id_part }}\right)=\left[1+e^{-\beta(d-T)}\right]^{-1}
$$

where:

$d$ - sum of absolute differences of the set of linear accelerations given by formula (2); size of the data set depends on the frequency of measurements and the velocity of measuring vehicle,

$\beta$ - parameter defining the function curve slope, where a high value implies stepwise behaviour of the function,

$T$ - parameter defining the function curve shift, considers the vehicle's free vibrations.

$$
d_{i d_{-} \text {part }}=\sum_{j}\left|a_{j}^{y}-a_{j-1}^{y}\right|
$$

where:

$a^{y}$ - recorded vertically oriented linear accelerations assigned to the given road section.

The behaviour of the function of road pavement condition description for the given input data and the pre-assumed values of parameters $\beta$ and $T$ has been illustrated in Fig. 3.

The cut-off threshold for free vibrations of vehicle depends on the vehicle type, and it was calculated as a mean value of the set of recorded linear accelerations for the vehicle types pre-defined in the S-mileSys system as they stopped with the engine on.

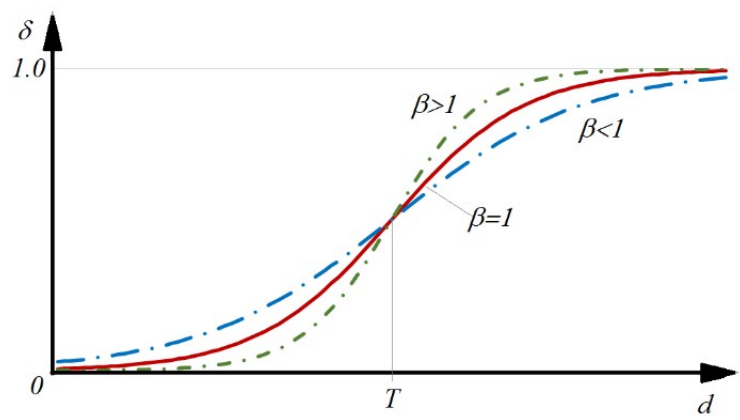

Fig. 3. Behaviour of the pavement condition description function.

Fig. 4 provides a graphical interpretation of the recorded linear accelerations along with the estimated index of road pavement condition description for two roads of considerably different technical condition. Section 1 is a fragment of road classified as critically in need of immediate repair works, while section 2 is a road fragment where the surface course of the pavement has just been replaced. The parameter which defines the slope of the curve of function $\beta$ depends on the driver's driving style and the vehicle type. Based on analyses performed over more than $2,500 \mathrm{~km}$ of routes covered by different drivers under different traffic conditions, a constant value was assumed for parameter $\beta$, adjusted depending on the vehicle type.

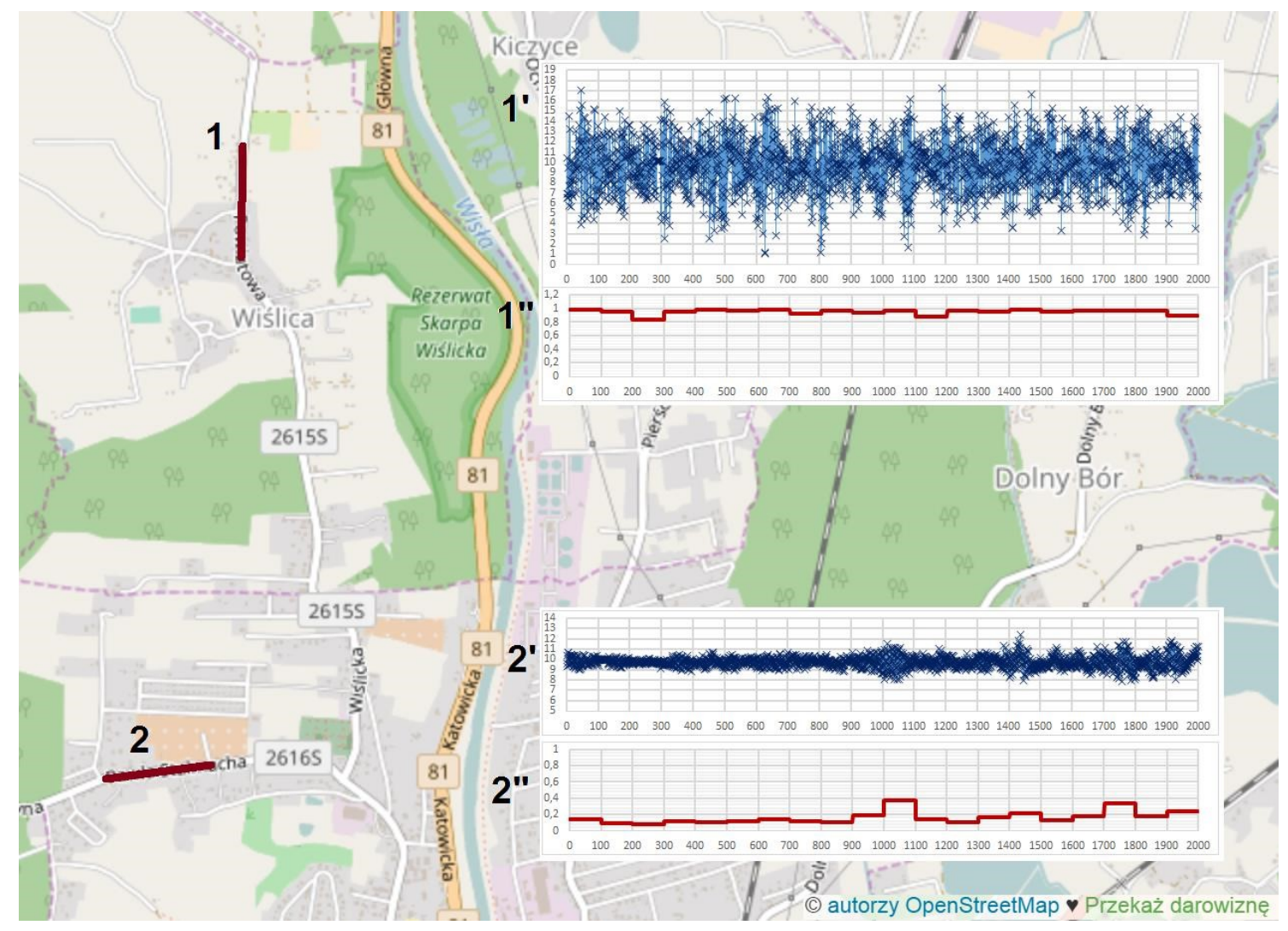

Fig. 4. Visualisation of the recorded linear accelerations along with estimated values of the pavement condition assessment index 
According to the S-mile project assumptions, vehicles of transport companies traversing the road network acquire data which subsequently enable the pavement condition assessment index to be established. Many times vehicles travelling across the road network use the same route sections, which means that the pavement condition assessment index for individual parts is estimated multiple times. Consequently, the value of the pavement condition assessment index for multiple transfers over the given part is calculated as an arithmetic mean based on indices established for the given time horizon of $D$. However, when a defect occurs and a repair procedure is conducted, calculation of the mean pavement condition assessment index is encumbered with an error. With regard to the foregoing, it was proposed that mean values of pavement condition assessment indices should be calculated for the two given time horizons of $S$ and $L$ :

$$
\begin{gathered}
\bar{\delta}_{i d \_p a r t}^{S}=\sum_{i=1}^{n_{S}} \delta_{i d \_p a r t}^{i} / n_{S} \\
\bar{\delta}_{\text {id_part }}^{L}=\sum_{i=1}^{n_{L}} \delta_{i d_{-} \text {part }}^{i} /\left(n_{L}-n_{S}+1\right)
\end{gathered}
$$

If relationship (5) is fulfilled, the mean value of the road pavement condition assessment index is calculated in accordance with relationship (6).

$$
\begin{gathered}
\bar{\delta}_{i d_{-} \text {part }}=\sum_{i=1}^{n} \delta_{i d_{-} \text {part }}^{i} / n \\
\left|\bar{\delta}_{\text {id_part }}^{L}-\bar{\delta}_{\text {id_part }}^{S}\right|<0.12
\end{gathered}
$$

A failure to fulfil relationship (5) determines what is referred to as a major change to the pavement condition assessment index. Where this is the case, the mean value of the pavement condition assessment index is calculated in accordance with equation (3). The pre-defined values of time horizons depend on the number of measurement results obtained while the S-mileSys platform is in operation. Therefore, time horizon $D$ was assumed to equal 30 days until the current date, while the short time horizon of $S$ was assumed to correspond to one day and at least 10 measurements, and the long time horizon was set at one week and 100 measurements at the least

\section{Assessment of the measurement method proposed}

In order to verify the RCT tool's operating efficiency and to check whether the estimated road pavement condition assessment indices were correct, the measurement method was verified in two ways. The first verification consisted in selecting road sections of specific technical class as per the applicable guidelines [20], followed by the road pavement diagnostics procedure performed in these sections using the tool in question. The choice of road sections was made in a breakdown into: new or rebuilt pavement (A), resurfaced pavement with occasional surface defects (B), pavement showing defects in need of planned repair (C) and pavement showing defects in need or immediate or major repair (D). The routing of measurement sections was described using GPS coordinates, after which they were divided into 10-metre parts. Selected measurement sections included fragments of roads of the following categories: regional, district and communal, all of which were located within borders of the Cieszyn administrative district.

Table 1 summarises the results obtained by means of

\begin{tabular}{|c|c|c|c|c|c|}
\hline 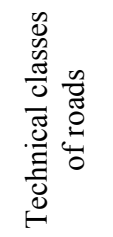 & 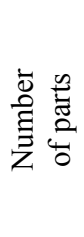 & 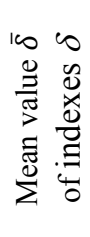 & 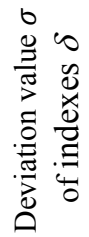 & $\begin{array}{c}0 \\
m \mid N \\
1 \\
100\end{array}$ & $\begin{array}{c}0 \\
m \text { IN } \\
+ \\
100\end{array}$ \\
\hline Class A & 163 & 0.099 & 0.054 & 0.019 & 0.179 \\
\hline Class B & 638 & 0.396 & 0.124 & 0.209 & 0.582 \\
\hline Class C & 371 & 0.702 & 0.071 & 0.596 & 0.808 \\
\hline Class D & 95 & 0.901 & 0.057 & 0.816 & 0.987 \\
\hline
\end{tabular}
the RCT measuring tool in a breakdown into technical classes of road pavement condition.

Table 1. RCT tool assessment - first method of verification

The results imply that the estimated pavement condition assessment indices assumed values representing different ranges depending on the analysed technical road condition class. The mean index value obtained for new roads was 0.1 , while for roads of poor technical condition - 0.9. Bearing in mind the mean value, the standard deviation as well as the normal distribution of occurrence of index values for the given class, one can establish disjoint ranges $\left(\bar{\delta}-\frac{3}{2} \sigma ; \bar{\delta}+\frac{3}{2} \sigma\right)$ defining the road pavement condition assessment indices assigned to the currently applicable technical classes. Thus, for the assumed range, at least $86.6 \%$ of the estimated index values obtained by means of the RCT tool matched the appropriate technical class.

The second method used to verify the RCT tool consisted in performing the road pavement diagnostics procedure and comparing its results with the assessment conducted by the road infrastructure administrator. The data set for which the comparison was performed was based on measurements conducted in national road DK1, from kilometre $560.045 \mathrm{~km}$ to kilometre $598.502 \mathrm{~km}$, i.e. between Tychy and Bielsko-Biała, excluding road sections cutting through territories of these municipalities. However, on account of the granulation of the available data determining technical road classes, the measure of general pavement condition assessment established by the General Directorate of National Roads and Motorways (GDDKiA) [20] with 1-kilometre graduation was assumed for the sake of the verification. This measure is expressed by three decision-making levels: desirable (D), warning (W) and critical (C).

Table 2 defines the numbers of individual road pavement condition assessment indices $N$ aggregated into 0.2 intervals within the range from 0 to 1 for the selected sections of the route connecting Tychy and Bielsko-Biała. 
Table 2. RCT tool assessment - second method of verification

\begin{tabular}{|c|c|c|c|c|c|c|}
\hline \multirow{2}{*}{ 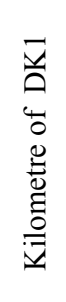 } & \multirow{2}{*}{ 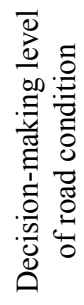 } & \multicolumn{5}{|c|}{$\begin{array}{l}\text { The number of aggregate } \\
\text { indicators for below ranges }\end{array}$} \\
\hline & & $\begin{array}{l}\text { Na } \\
0 \\
1 \\
0 \\
0\end{array}$ & $\begin{array}{l}+ \\
0 \\
1 \\
\\
0\end{array}$ & $\begin{array}{l}0 \\
0 \\
1 \\
\dot{1} \\
\dot{0}\end{array}$ & $\begin{array}{l}\infty \\
0 \\
1 \\
0 \\
0 \\
0\end{array}$ & $\begin{array}{c}0 \\
\dot{1} \\
1 \\
\infty \\
0\end{array}$ \\
\hline 578 & W & 0 & 2 & 17 & 68 & 13 \\
\hline 579 & D & 7 & 45 & 24 & 19 & 5 \\
\hline 580 & $\mathrm{C}$ & 0 & 3 & 9 & 21 & 67 \\
\hline 581 & $\mathrm{D}$ & 5 & 59 & 31 & 4 & 1 \\
\hline 582 & D & 8 & 42 & 38 & 7 & 5 \\
\hline 583 & W & 3 & 5 & 12 & 59 & 21 \\
\hline 584 & $\mathrm{C}$ & 0 & 2 & 9 & 35 & 54 \\
\hline 585 & C & 1 & 4 & 7 & 27 & 61 \\
\hline 586 & $\mathrm{~W}$ & 0 & 2 & 8 & 74 & 16 \\
\hline
\end{tabular}

The predominance of the number of occurrences of estimated road pavement condition assessment indices for the assumed ranges corresponds to the aforementioned general road pavement condition assessment. Thus, for the general assessment of the desirable condition, the highest number was found in the range of $0.2-0.6$, while for the warning and critical condition assessments - in the ranges of $0.6-0.8$ and $0.8-1.0$ respectively.

\section{Conclusions}

The RCT tool enables continuous road pavement diagnostics, identification and recording of the current technical condition of road infrastructure as well as of volume and distribution of current freight transport traffic. It is the responsibility of local authorities and road administration bodies to support the process of decision making in the scope of management and maintenance of road pavement by indicating road sections that require more detailed assessment as well as sections in need of immediate or planned repairs.

The results discussed for the selected road sections confirm the potential of the measurement method proposed. The solution in question requires no highly advanced measuring equipment and causes no nuisance to other participants of road traffic. It functions on a continuous basis while transport services are performed within city or agglomeration borders, and yet it needs no intervention from the driver. Once the transport process is completed, the data recorded on the way are immediately sent to the system's server where road pavement condition assessment indices are calculated automatically.

Currently works, about RCT tool consists in evaluating the repeatability of the results obtained from the measurements carried out by various types of a vehicles on the road network in various technical condition. A part of studies were presented in [21]. Future work will concern determining the type and its size of distresses based on recorded signal of the vehicle dynamics which is captured with the applied measurement tool.

The present solution has been financed from the means of the National Centre for Research and Development as a part of the international project within the scope of ERA-NET Transport III Sustainable Logistics and Supply Chains Programme "Smart platform to integrate different freight transport means, manage and foster first and last mile in supply chains (S-mile)".

\section{References}

1. D. Hale, J. Hale, J. Lindly, R. Sharpe, D. Turner, AISCE Report 09-1001 (2009)

2. E. Baran, D. Angell, S. Earl, S. Walsh, Australian Road Research Board (ARRB) Conference, 14.6 (1988)

3. D. Sybilski, T. Mechowski, P. Harasim, Roads and Bridges-Drogi i Mosty, 6.2 (2007)

4. A. Zofka, J. Sudyka, M. Maliszewski, P. Harasim, D. Sybilski, TRANS RES B, 2457 (2014)

5. M. Graczyk, L. Krysiński, Ł. Topczewski, J. Sudyka, TRANSP RES PROC, 14 (2016)

6. K. McGhee, TRANS RES B, 334 (2004).

7. M. Rogers, B. Enright, Highway engineering (2016).

8. M. Wasilewska, W. Gardziejczyk, P. Gierasimiuk, TRANSP RES PROC, 14 (2016)

9. J. Hall, K. Smith, L. Titus-Glover, J. Wambold, T. Yager, Z. Rado, NCHRP Report, 01-43 (2009)

10. J. El Khoury, B. Akle, S. Katicha, A. Ghaddar, M. Daou, INT J PAVEMENT ENG, 15.4 (2014)

11. Henry J. J.: NCHRP Synthesis 291. Transportation Research Board, (2000)

12. Flintsch G. W., Huang M., McGhee K.: Journal ASTM International, 2.9 (2005)

13. S. Varadharajan, S. Jose, K. Sharma, L. Wander, C. Mertz. Applications of Computer Vision - IEEE Winter Conference (2014)

14. S. Chambon, J. Moliard, International Journal of Geophysics, 2011 (2011)

15. Technical specification: Pavement Monitoring by the ARGUS® Survey System by TÜV Rheinland Schniering GmbH. Available schniering.com (2017)

16. Ghose, P. Biswas, C. Bhaumik, M. Sharma, A. Pal, A. Jha, Pervasive Computing and Communications Workshops - IEEE International Conference (2012).

17. M. Agostinacchio, D. Ciampa, S. Olita, EUR TRANSP RES REV, 6.3 (2014)

18. G. Leduc, Working Papers on Energy, Transport and Climate Change, 47967 (2008)

19. J. Żak, B. Galińska, Advances in Intelligent Systems and Computing, 572 (2016)

20. Regulation of General Director of National Roads and Motorways on the diagnosis of the road pavement condition and its elements (in Polish), 34 (2015)

21. Staniek M.: IOP Conf. Ser.: Mater. Sci. Eng. 356.012021 (2018) 